\title{
INTERACTIONS OF DNA WITH ZORBAMYCIN, PHLEOMYCIN AND BLEOMYCIN; ULTRAVIOLET ABSORPTION AND CIRCULAR DICHROISM MEASUREMENTS
}

\author{
William C. Krueger,* Loraine M. Pschigoda and Fritz Reusser \\ Research Laboratories, The Upjohn Company, \\ Kalamazoo, Mich. 49001, U.S. A. \\ (Received for publication April 16, 1973)
}

\begin{abstract}
Mixtures of DNA with the three antibiotics zorbamycin, phleomycin or bleomycin were analyzed by UV and circular dichroism measurements. The results indicate that a physical interaction between isolated DNA and each of the three antibiotics occurs and that these antibiotics definitely bind to DNA.
\end{abstract}

Zorbamycin is a broad spectrum antibacterial and antifungal agent and is related to the phleomycins and bleomycins. Zorbamycin was found to induce rapid degradation of the DNA and RNA fractions in bacterial cells ${ }^{11}$. DNA degradation is initiated first and is closely followed by the degradation of RNA. No degradation of isolated DNA is observed in the presence of zorbamycin. DNA and RNA syntheses are not impaired in cell-free systems. Since the initial effect of the antibiotic is expressed at the level of the DNA fraction, it was concluded that zorbamycin somehow induces a change in the structure or function of the cellular DNA fraction which leads to a rapid breakdown of this fraction. No evidence for any direct interaction of isolated DNA with zorbamycin could be demonstrated by a variety of parameters. We now wish to present ultraviolet (UV) and circular dichroism (CD) measurements which indicate that a physical interaction between isolated DNA and zorbamycin does indeed occur. For comparative purposes, UV and CD data obtained with phleomycin- and bleomycin-DNA mixtures are also included. Phleomycin and bleomycin have been shown to interact with DNA by techniques other than UV and $\mathrm{CD}^{2}$.

\section{Materials and Methods}

Calf thymus DNA was purchased from Worthington Biochemicals. Bleomycin, $\mathrm{Cu}^{2+}$-free, was purchased from Nippon Kayaku, Co., Japan; Phleomycin-Cu ${ }^{2+}$ complex was obtained from Bristol Laboratories, Inc. The structures of these antibiotics have been published recently ${ }^{2,3,4)}$. Their molecular weights amount to approximately 1,540. The structure of zorbamycin is unknown but its molecular weight is estimated to be similar to phleomycin and bleomycin. Direct comparisons of their effects on an equal weight basis is thus permissible. The name zorbamycin applies to the $\mathrm{Cu}^{2+}$-containing complex of the antibiotic.

Circular dichroism (CD) data were obtained on a Cary 60 spectropolarimeter interfaced with an IBM 1800 computer. The instrument was calibrated with 10 -camphorsulfonic acid ${ }^{4,8)}$. Ultraviolet absoprtion (UV) data were also obtained on the Cary 60 (simultaneously with the $\mathrm{CD}$ ) from a record of the dynode voltage of the CD phototube. The UV difference spectra were obtained on a Cary 15 spectrophotometer. The vertical bars above the $\mathrm{CD}$ curves represent the estimated error of the measurements. The false rotation of the Cary 60 was checked with dichromate solution and found to be about 0.001 degree at an absorbance of about 2.5 .

All compounds were dissolved in water. The exact compositions of the reaction mixtures are given in the Figure legends. The UV and CD characteristics of the compounds used in these studies

\footnotetext{
* to whom correspondence should be sent.
} 
are detailed in the following. The wavelength maxima are expressed in millimicrons followed by the intensity in terms of specific quantities: UV, absorptivity; $\mathrm{CD}$, specific ellipticity (degrees ellipticity divided by the path length in $\mathrm{dm}$ and the concentration in $\mathrm{g} / \mathrm{cm}^{3}$ ).

Calf Thymus DNA. UV: $258 \mathrm{~m} \mu$ (15.5); CD: $218 \mathrm{~m} \mu$ (950), $245 \mathrm{~m} \mu(-2240), 275 \mathrm{~m} \mu$ (2080). Zorbamycin. UV: $244 \mathrm{~m} \mu(15.9), 298 \mathrm{~m} \mu(5.0)$; CD: $230 \mathrm{~m} \mu(-390), 253 \mathrm{~m} \mu(1470), 270 \mathrm{~m} \mu$ $\operatorname{sh}(8 \overline{80), 313 \mathrm{~m} \mu}(-760)$.

Phleomycin. UV: $244 \mathrm{~m} \mu$ (13.2), $298 \mathrm{~m} \mu$ (4.6); CD: $230 \mathrm{~m} \mu$ (0), $251 \mathrm{~m} \mu$ (1270), $272 \mathrm{~m} \mu$ $\operatorname{sh}(4 \overline{70), 312 \mathrm{~m} \mu}(-700)$.

Bleomycin. UV: $235 \mathrm{~m} \mu \mathrm{sh}(14.2), 291 \mathrm{~m} \mu(9.5)$; CD: $248 \mathrm{~m} \mu(-200), 285 \mathrm{~m} \mu(470)$.

\section{Results and Discussion}

The individual CD and UV absorption curves obtained with calf thymus DNA, zorbamycin, phleomycin or bleomycin are shown in Fig. 1. DNA has CD maxima at 275, 245 (negative) and $218 \mathrm{~m} \mu$ and a UV maximum at $258 \mathrm{~m} \mu$. Zorbamycin and phleomycin possess similar UV and CD characteristics in that both compounds show a broad UV band near $300 \mathrm{~m} \mu$, a well defined UV maximum at $244 \mathrm{~m} \mu$ and $\mathrm{CD}$ bands near 315 (negative), 270 (shoulder) and $250 \mathrm{~m} \mu$. The major difference between the $\mathrm{CD}$ curves of zorbamycin and phleomycin is observed at $230 \mathrm{~m} \mu$ where the ellipticity values are negative for zorbamycin and zero or positive for phleomycin. This difference is reproducible and significant as it is in excess of the estimated experimental error. The UV and $\mathrm{CD}$ spectra for bleomycin differ substantially from those of either zorbamycin or phleomycin. The $\mathrm{CD}$ spectrum of bleomycin is dominated by a large positive band at $285 \mathrm{~m} \mu$ which is probably due to the same electronic transition(s) responsible for the UV maximum at $290 \mathrm{~m} \mu$. In addition bleomycin shows a slight shoulder near $240 \mathrm{~m} \mu$ in the UV spectrum and a weak negative band near $250 \mathrm{~m} \mu$ in the $\mathrm{CD}$ spectrum. The position of this weak $\mathrm{CD}$ band must be considered somewhat uncertain due to the relatively large uncertainty of the ellipticity values below $250 \mathrm{~m} \mu$.

That the UV and CD spectra of $\mathrm{Cu}^{2+}$-free bleomycin differ substantially from those of phleomycin and zorbamycin may be due to the effect of $\mathrm{Cu}^{2+}$-chelation on the spectra of phleomycin and

Fig. 1. CD $(-)$ and UV (--) spectra of: (A) calf thymus DNA (200 $\mu \mathrm{g} / \mathrm{ml}$ in $\mathrm{H}_{2} \mathrm{O}$ ); (B) zorbamycin; (C) phleomycin and (D) bleomycin. All antibiotic solutions were $80 \mu \mathrm{g} / \mathrm{ml}$ in $\mathrm{H}_{2} \mathrm{O}$.
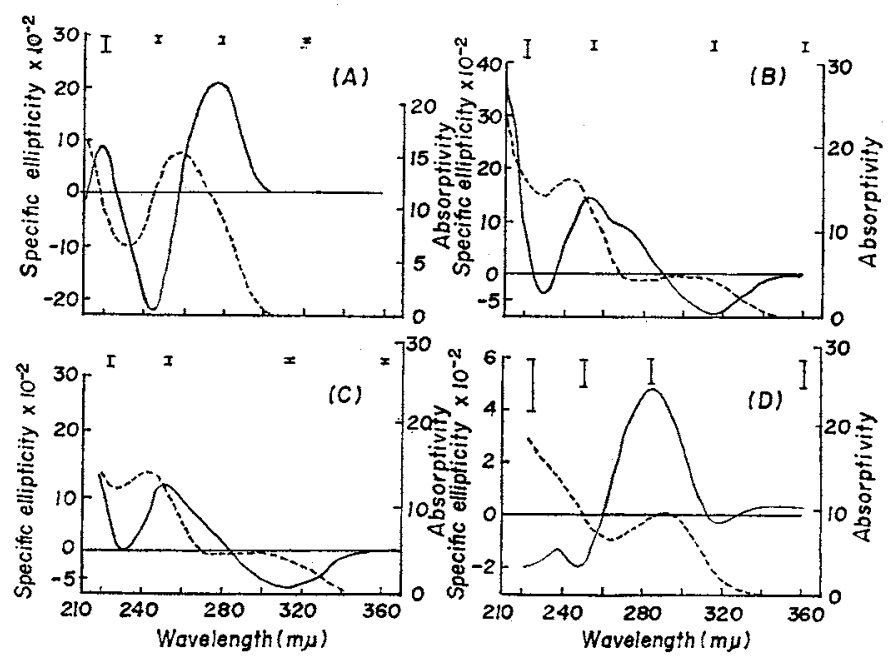

zorbamycin and/or to the chromophoric differences of the antibiotics. Thus, bleomycin contains a conjugated di-thiazole ring system while,phleomycin does not because of one less carbon-carbon double bond in one of the thiazole rings ${ }^{4}$. Nevertheless, the simplest interpretation of the DNA-antibiotic interaction studies described below is the same for all three antibiotics even though the UV and $\mathrm{CD}$ spectra of bleomycin differ significantly from those of phleomycin and zorbamycin. 
Fig. 2. CD (A) and UV (B) spectra of DNA (100 $\mu \mathrm{g} / \mathrm{ml})$ and zorbamycin $(40 \mu \mathrm{g} / \mathrm{ml})$ in $\mathrm{H}_{2} \mathrm{O}$, in separate cells (a) and the same cell (b).

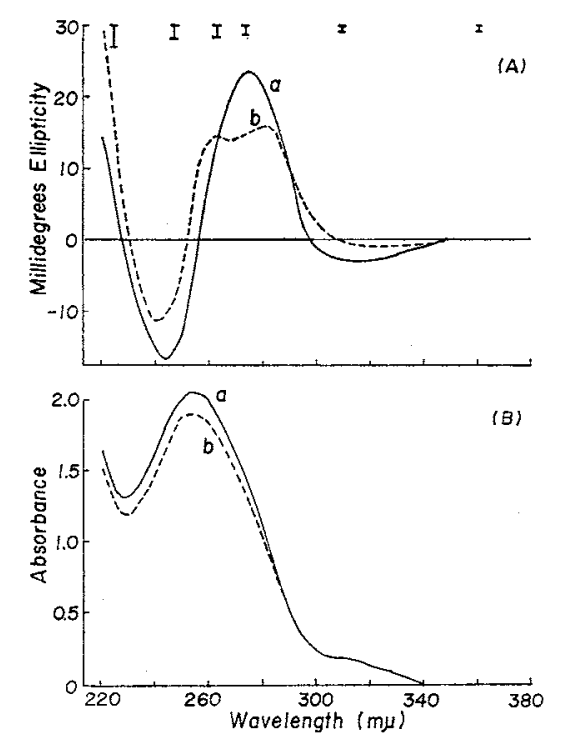

The CD and UV curves of DNA-zorbamycin mixtures are given in Fig. 2. The control curves (solid lines) were obtained with DNA and zorbamycin present in separate cells, each read against water as a blank and represent the sum of the curves shown in Figs. 1A and 1B. The dashed curves in Fig. 2 were obtained with zorbamycin and DNA mixed in a single cell.

It is evident that both spectra of the DNAzorbamycin mixtures differ significantly from the control spectra although the differences are more enhanced in the CD curve. This indicates that some interaction had occurred between DNA and zorbamycin upon mixing of the two components. However, the specific nature of the electronic transitions responsible for these spectral differences are difficult to define.

These differences are better visualized by the use of difference spectra. Difference UV and CD spectra were thus prepared with DNA mixed with either zorbamycin, phleomycin or bleomycin (Fig. 3). The curves represent the data obtained with DNA-antibiotic mixtures (baseline) subtracted from the data of DNA and antibiotic in separate cells (sample line). In the case of the DNAzorbamycin mixtures the difference spectra are thus equivalent to the differences between the two UV and CD spectra shown in Fig. 2. The spectral changes occurred instantly upon mixing of the components and remained stable for at least the duration of the time needed to record the spectra $(1 \sim 2$ hrs. $)$.

The difference $C D$ spectrum obtained with the DNA-zorbamycin mixture shows a shallow negative deflection from the baseline at approximately $300 \mathrm{~m} \mu$, a strong positive deflection at $270 \mathrm{~m} \mu$ 
and another negative deflection at $250 \mathrm{~m} \mu$. A similar CD difference spectrum was obtained with a mixture of DNA and phleomycin. The only distinct difference between the DNA-zorbamycin and DNA-phleomycin CD difference spectra resides in the intensity of the negative deflection at $250 \mathrm{~m} \mu$.

The CD difference spectrum obtained for the DNA-bleomycin mixture differs significantly from the ones obtained with either zorbamycin or phleomycin. A negative maximum is observed at $290 \mathrm{~m} \mu$ and a broad, shallow positive de lection with a maximum near $258 \mathrm{~m} \mu$.

The UV difference spectra of the three DNA-antibiotic mixtures are all quite similar as each shows a positive maximum near $258 \mathrm{~m} \mu$.

The interpretation of these CD and UV difference curves in terms of which of the two respective components of the mixtures contributes, and to what extent, toward the measured deflections is difficult. As DNA and antibiotics possess UV and CD absorption, both DNA and the antibiotics upon mixing and interaction with each other can contribute to the net observed difference spectra. Overlapping effects of neighboring absorption bands might have opposite signs and to some extent might cancel each other. We present here the simplest interpretation of the data obtained.

The $C D$ results suggest that the antibiotics bind to DNA without causing an extensive change in the secondary structure of the DNA helix. On the other hand a significant change in the rotational characteristics of the antibiotic transitions occurs as a result of asymmetric perturbations induced by the DNA molecule as the antibiotics bind to DNA. This interpretation is based on the observation that the CD maxima of the DNA-antibiotic difference spectra (Fig. 3) coincide somewhat more closely with the $\mathrm{CD}$ maxima for the antibiotics alone rather than with the $\mathrm{CD}$ maxima for DNA alone (Fig. 1). No positive or negative remnant contributions of DNA at the $275 \mathrm{~m} \mu$ or $245 \mathrm{~m} \mu$ regions are evident or necessary to interpret the difference curves. This interpretation is perhaps somwhat more clear in the case of the DNA-bleomycin CD difference spectrum.

The common feature of the UV difference spectra, a positive maximum near $258 \mathrm{~m} \mu$, indicates that the absorptivity of the UV band in this region has decreased. As the antibiotics alone possess low absorbance in this region of the UV spectrum, we may assume that the decrease of absorbance in the $258 \mathrm{~m} \mu$ region can be attributed to the DNA component present in the mixture. Such a decrease in absorbance on the part of the DNA indicates that, as a result of the interaction of the DNA with the antibiotics, the degree of regularity of the DNA helix increases somewhat.

Zorbamycin and phleomycin alone show a weak CD band at $570 \mathrm{~m} \mu$ (not shown in Fig. 1). Attempts to measure interactions between DNA and phleomycin at this band failed due to formation of precipitates upon mixing of DNA and antibiotic at antibiotic concentrations $(1 \mathrm{mg} / \mathrm{ml})$ sufficiently high to allow detection of the $570 \mathrm{~m} \mu$ band. No precipitation occurred with DNA-zorbamycin mixtures under the same conditions. However, the rotational characteristics of the $570 \mathrm{~m} \mu$ band in the CD spectrum did not change upon mixing of DNA with zorbamycin.

\section{References}

1) Reusser, F.: Mode of action of zorbamycin. J. Bact. $108: 30 \sim 37,1971$

2) Suzuki, H.; K. Nagal, H. Yamaki, N. TANaka \& H. Umezawa: On the mechanism of action of bleomycin: scission of DNA strands in vitro and in vivo. J. Antibiotics $22: 446 \sim 448,1969$. NAGAI, K.; H. SUZuki, N. TANAKA \& H. UMEZAwa: Decrease of melting temperature and single strand scission of DNA by bleomycin in the presence of hydrogen peroxide. J. Antibiotics $22: 524 \sim 628,1969$. NAGAI, K.; H. SuZuki, N. Tanaka \& H. Umezawa: Decrease of melting temperature and single strand scission of DNA by bleomycin in the presence of 2-mercaptoethanol. J. Antibiotics $22: 569 \sim 573,1969$. NAGAI, 
K.; H. Yamaki, H. Suzuki, N. Tanaka \& H. Umezawa: The combined effects of bleomycin and sulfhydryl compounds on the thermal denaturation of DNA. Biochim. Biophys. Acta $179: 165 \sim 171$, 1969. Suzuki, H.; K. NagaI, E. Akutsu, H. Yamaki, N. Tanaka \& H. UMeZawa: On the mechanism of action of bleomycin. Strand scission of DNA caused by bleomycin and its binding to DNA in vitro. J. Antibiotics $23: 473 \sim 480,1970$.

3) Omoto, S.; T. Takita, K. Maeda, H. Umezawa \& S. Umezawa: Chemistry of bleomycin. ViII. The structure of the sugar moiety of bleomycin $A_{2}$. J. Antibiotics $25: 752 \sim 754,1972$

4) Takita, T.; Y. Muraoka, T. Yoshoka, A. Fujir, K. Maeda \& H. Umezawa: The chemistry of bleomycin. IX. The structures of bleomycin and phleomycin. J. Antibiotics $25: 755 \sim 758,1972$

5) Krueger, W.C. \& L. M. Pschigoda: Circular dichroism calibration by Kramers-Krönig transform methods. Ana1. Chem. $43: 675 \sim 677,1971$ 